\title{
A Large-Scale Detection, Identification and Quantification of Target Metabolites using dMRM- MS Combined with Transcriptome of Two Rheum Species Focused on Anthraquinone and Flavonids Biosynthesis
}

sha chen ( $\square$ schen@icmm.ac.cn )

China Academy of Chinese Medical Sciences Institute of Chinese Materia Medica https://orcid.org/0000-0003-1387-9133

Jing Liu

China Academy of Chinese Medical Sciences Institute of Chinese Materia Medica Liang Leng

China Academy of Chinese Medical Sciences Institute of Chinese Materia Medica Han Gao

China Academy of Chinese Medical Sciences Institute of Chinese Materia Medica

Wei Yang

China Academy of Chinese Medical Sciences Institute of Chinese Materia Medica An Liu

China Academy of Chinese Medical Sciences Institute of Chinese Materia Medica

Research article

Keywords: Rheum, RNA-seq, transcriptome, Anthraquinone, pharmacological metabolite

Posted Date: November 19th, 2019

DOI: https://doi.org/10.21203/rs.2.17449/v1

License: (c) (i) This work is licensed under a Creative Commons Attribution 4.0 International License.

Read Full License 


\section{Abstract}

Background Rheum emodi ( R. emodi ) is a perennial herb as well as an important medicinal plant. The main active compounds in Rhubarb are anthraquinones, but also contains flavonoids. As a class of important active components in rhubarb, anthraquinones have many pharmacological effects. However, due to the lack of rhubarb genome, it is difficult to mine functional genes involved in the biosynthesis of anthraquinones in rhubarb. Results Based on the a large-scale quantification of target metabolites combined with the transcriptome data of Rheum Palmatum L . (RPL) and Rheum Officinale Bails (ROB), in this study we aimed to firstly mine out anthrquinones and other pharmacological metabolites in rhubarb, then search genes which may related to the biosynthesis of anthraquinones and other metabolites. Via dynamic-multiple reaction monitoring (dMRM) of triple quadrupole mass spectrometry (QqQ-MS) technical means, 62 compounds including 21 anthraquinones, 17 flavonoids, 6 stilbenes, 12 gallate esters, 3 tannins, and 3 others were simultaneous qualitatively and quantitatively assessed. Furthermore, a total of 180,689 transcripts and 90,242 unigenes were generated by the de novo transcriptome assembly. Down-stream bioinformatic analyses annotated the unigenes with BLAST against seven public databases including KEGG and KOG. GO and KEGG pathway analyses further characterized the unigenes, resulting in 21,691 unigenes with metabolic pathway annotations. There were 121 genes annotated in the flavonoid pathways, and 38 of them showed a significant difference in expression level between the RPL and ROB. According to the difference in the content of emodin and physcion in the two kinds of rhubarb and the expression level of key enzyme genes, we further search 7 differentially expressed genes annotated with the activity of caffeoyl coenzyme methyltransferase. RTqPCR results showed that the expression of the cluster-14354.38156 in the ROB was approximately 14fold higher than that observed in the RPL, which may participate in the process of 0-methylation from emodin to physcion. Conclusion Combining metabolome and transcriptome analysis, a key enzyme gene was identified. This study supplied a useful resource for further study of metabolite in rhubarb.

\section{Background}

Rheum emodi (R. emodi), also called "DaHuang", polygonaceae, is a species of rhubarb rich repository of pharmaceutically important secondary metabolite constituents of the anthraquinones group [1]. In ancient China and Western countries, rhubarb has mainly been used to clear away heat and assist with defecatione. China has been the major global producer of rhubarb. In addition, it has been a great demand for rhubarb in Europe owing to the local eating habits. Rhubarb has been used as an effective, short-lived, and painless cathartic with important therapeutic effects [2]. The usage of the roots and rhizomes of rhubarb was frequently featured in traditional Chinese pharmacopeia, e.g., Shennong Ben Cao Jing and Ben Cao Gang Mu. There are three identified species of rhubarb in Chinese Pharmacopoeia, of which the two wildly mixed used in clinical medicine namely $R$. palmatum $L$. (RPL), and $R$. officinales Bail (ROB), and there were lacking reports about the differences of the pharmaceutically metabolites between them [3]. However, the main active compounds in Rhubarb are anthraquinones [4], but also contains flavonoids. 
The main anthraquinone derivatives in rhubarb are mostly emodin anthraquinone. Currently, there are about more than 30 anthraquinone compounds in rhubarb, including emodin, aloe emodin and physcion [5]. As to the same skeleton construction, different structural modifications endow metabolites with different pharmacological effects [6]. Anthraquinone compounds in rhubarb belong to aromatic polyketone, which is obtained by the pathway of acetic acid biosynthesis. In the initial synthesis stage, acetyl coenzyme-A was taken as the initial unit and malonyl coenzyme-A as the extension unit, and the benzene ring structure was formed under the action of polyketide synthase [7]. Under the catalysis of plant specific type III polyketo compound synthase, the precursor of free anthraquinone -- anthrone, was generated through hydroxy aldehyde reaction, enolization reaction, oxidation reaction and decarboxylation reaction. Then free anthraquinone was generated into tuberculosis type anthraquinone under the action of glycosyltransferase $[8,9]$. Some studies have shown that methyltransferase catalyzes the hydroxyl methylation of emodin 3 to form physcion [10]. Similar to this pathway is the biosynthesis of flavonoids, the initial synthesis of flavonoids was also conducted under the action of polyketide synthase with malonyl coenzyme-A as the extension unit [11-14]. Then, chalcone, a precursor of flavonoids, was synthesized by plant specific type III polyketide synthase through oxidation reaction, hydroxy aldehyde reaction, synthesis and isomerism reaction. The free flavonoids were produced from chalcone catalyzed by chalcone isomerase, and then flavonoid glycosides were further produced by glycosyltransferase.

Although biosynthesis of anthraquinones is aim of some former studies in rhubarb [11, 15-17], the biosynthesis pathway of anthraquinones is not as clear as that of flavonoids. And the results from investigation of the metabolic pathway of anthraquinones are also limited in the currently available databases. The mechanism of skeleton formation of anthraquinones remains to be explored $[16,18]$. Therefore, the key enzyme genes involved in the biosynthesis of anthraquinones were explored by combining the biosynthesis information of flavonoids. Besides, due to the lack of rhubarb genome, it is difficult to search for functional genes. In the post-genomic data era, integrated analysis of comprehensive gene expression (transcriptome) and metabolic profiling (metabolomics) data has successfully applied in plant functional research $[19,20]$. Thus, in this study, we supplied both metabolome and transcriptome data of Rheum Palmatum L. (RPL) and Rheum Officinale Bails (ROB) as resources for future research. At the same time, we searched the genes that might be involved in the biosynthesis of anthraquinones.

\section{Results}

\section{Mass Conditions}

The metabolic analysis of Rheum Officinales Bails (ROB) and Rheum Palmatum L. (RPL) leaves were optimized by comparing the performance of several candidate elution systems, based on previous references data[21]. The acidity of the elution system is the most important factor for the analysis of metabolism. Therefore, different elution acidities with $0.1 \%, 0.2 \%, 0.5 \%$, and $0.8 \%$ formic acid were evaluated by comparing the obtained target compounds and resolution of all the metabolism. Finally, $0.2 \%$ formic acid exhibited the best performance. The optimized HPLC and electrospray ionization-QqQ- 
MS (ESI-QqQ-MS) conditions were as follows: the solvent system, eluent A was milli-Q water containing $0.2 \%$ formic acid, and eluent $B$ which was acetonitrile. All samples were analyzed using an Eclipse-Plus $\mathrm{C}_{18}$ column $(2.1 \times 50 \mathrm{~mm}, 1.8 \mu \mathrm{m})$ at $30^{\circ} \mathrm{C}$ with a linear elution gradient protocol of $0-1.5 \mathrm{~min}, 95 \% \mathrm{~B}, 1.5-3$ min, 5-24\% B, 3-5min, 24-25\% B, 5-11 min, 25-66\% B; $11-16$ min, 66\% B; 16-17 min, and 66-100\% B, with a flow rate of $0.2 \mathrm{~mL} / \mathrm{min}$. Under these conditions, the metabolites of rhubarb leaves were completely eluted with resolving powder best target (Additional file 1).

\section{Identification of rhubarb leaves metabolites based on ESI-Q-TOF-MS/MS}

The identification of metabolites in conjunction with the ESI-Q-TOF-MS, both positive and negative ESI modes, were used to determine fragment ion information of metabolites. Information including mass spectra (in $\mathrm{PI}, \mathrm{NI}, \mathrm{NI}^{-\mathrm{MS}^{2}}$, and $\mathrm{PI}-\mathrm{MS}^{2}$ ), ultraviolet-vis spectra and retention time on $\mathrm{C}_{18}$ column are list in table 1. Based on the dissociation rules of different metabolites in rhubarb leaves, the precise molecular weights were confirmed after eliminating false positive rates. Firstly, the MS/MS behavior was resolved by fragment characteristics. Based on the combination of data available on the mass-bank and previous reference data [1, 22], 62 compounds were identified including 21 anthraquinones, 17 flavonoids, 6 trimethyltin, 12 gallates, 3 tannic acid, and 3 others. Anthraquinones and flavonoids are the main pharmacologically active ingredients of rhubarb. The detected metabolites are modified at one or several positions by methylation, glycosylation, finally endow them with different activities. The metabolic network diagram of rhubarb flavonoids and anthraquinone is well constructed, as showed in Figure 1. The recovery index of characteristic compounds was used to measure the accuracy of the method, showing good repeatability.

\section{A Large-scale Detection, Identification and Quantification of Target Metabolites using dMRM-MS/MS}

A large-scale quantification method was carried out constructing a tandem mass spectrometry (MS2) spectral tag library from detection of target metabolites using paired ions. A dynamic multiple reactions monitoring (MRM) mode under unit mass-resolution was used for simultaneous quantitative measured all the detected metabolites. It is the first time, simultaneous quantitatively identified and qualitatively

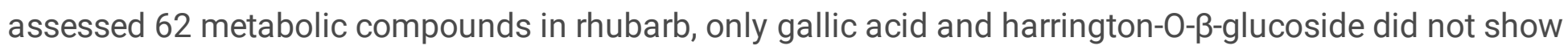
significant differences between the two species. The remaining 60 compounds exhibited significant differences $(P<0.05)$. The contents of aloe emodin, rhein, and emodin-0-(6-0-acetyl)-glucoside in RPL leaves were significantly higher than those observed in the ROB leaves. In addition, the contents of chrysophanol, emodin and methyl ether were significantly higher than those reported in ROB. Moreover, the contents of chrysophanol-(6-0-acetyl)-glucoside and 43 other substances in the RPL were significantly lower than those measured in the ROB. 
The contents of 8-0-methyl chrysophanol, chrysophanol, and physcion in the RPL were significantly lower than those observed in the ROB. Furthermore, the contents of three free anthraquinones in the RPL were significantly higher than those reported in ROB. Of the 14 binding anthraquinones, the content in the RPL was significantly lower than that detected in ROB, whereas the content of the other six in the ROB was significantly higher than in the RPL.

Principal component analysis was used to examine the quantitative data of the anthraquinone compounds in two kinds of rhubarb. The variance contribution rate of the first eigen-value was $92.61 \%$, the second eigen-value was $3.62 \%$, and the accumulative contribution rate of the first two factors was 96.23\%. The six replicates of ROB and RPL leaves were clustered on the left and right sides of PC1, respectively, indicating a good correlation between biological duplication and a significant difference between the two kinds of rhubarb (Figure 2A). There was a significant difference in the content of anthraquinones between the two rhubarb species (Figure 2B).

\section{Method validation}

The calibration curves showed good linearity for all the available standards. The standard solutions were detected through chromatography until the signal-to-noise ratio corresponded to 3 and 10 . The corresponding concentrations at these signal-to-noise ratios were defined as the limit of detection and limit of quantitation, respectively. The precision of metabolic quantification was studied by examining the repeatability and intermediate precision for all the compounds.

\section{Sequencing and de novo transcriptome assembly}

RNA was sequenced using Illumina paired-end sequencing technology. A total of 193,873,248 Illumina raw reads were obtained (Table 2). After removing adaptor sequences, ambiguous nucleotides and lowquality sequences, approximately 185 million clean reads were kept for the subsequent analysis. These reads were then assembled de novo using Trinity [23]. After eliminating repeated sequences and sequences $<200 \mathrm{bp}$, the assembly of clean reads resulted in 180, 689 transcripts and 90,242 unigenes. These unigenes ranges from 201 to $14,669 \mathrm{bp}$, with a mean length of 1,052 bp, median length of $706 \mathrm{bp}$ and N50 length of 1,556 bp (Additional file 2).

\section{Annotation of unigenes}

To access the quality of these assembled unigenes, a homology search in $\mathrm{Nr}$ database was conducted for all unigenes using the BLAST program [24] and a cutoff E-value $<10^{-5}$. The best aligning results were selected to annotate the unigenes. The results showed that $56 \%$ of the aligned sequences $(50,558)$ 
exhibited significant homology with entries in the $\mathrm{Nr}$ database (E-value $<10^{-5}$ ), while $>75 \%$ of these matched sequences showed an E-value $<10^{-15}$ (Additional file 3). Based on the BLAST similarity distribution, $32.5 \%$ of the aligned sequences exhibited alignment identities $>80 \%$ (Additional file 3 ). We screened these 90,242 non-redundant unigenes for similarity in seven public databases (i.e., Nr, Nt, SwissProt, KEGG, GO, KOG, and Pfam). The annotation results showed that $56.05 \%(50,588$ unigenes) had hits in the $\mathrm{Nr}$ database, followed by the Swiss-Prot database (45.15\%, 40,753 unigenes). In total, there were 57,239 unigenes $(63.42 \%)$ annotated in at least one of these seven databases, with 8,819 unigenes (9.77\%) appearing in all seven databases including annotated in Nr, Nt, KEGG Orthology, SwissProt, Pfam, GO, Databases, at least one database, as well as total unigens.

\section{Functional classifications of unigenes}

GO (Gene Ontology) was used to classify the functions of the predicted rhubarb unigenes [25]. In total, 39,567 unigenes with BLAST matches to known proteins were classified into 56 functional groups in three main categories (i.e., biological process, cellular component, and molecular function) using 1660 functional terms (Figure $3 \mathrm{~A}$ ). The majority of the unigenes were assigned to the categories of biological processes $(488,916)$, followed by cellular components $(145,217)$ and molecular functions $(98,389)$. Within biological processes, metabolic process $(242,663)$ and cellular process $(108,192)$ were the most dominant terms. Under the category of cellular components, cell part $(44,594)$ and organelle $(33,420)$ had the highest representation. For molecular functions, the most represented were binding $(72,789)$ and catalytic activities $(18,096)$.

All unigenes were aligned with the $(K O G)$ database in which orthologous gene products were classified to classify possible functions. A total of 16,395 unigenes were clustered into $25 \mathrm{KOG}$ classifications (Figure 3B, Table 2), in which the cluster of translation, ribosomal structure and biogenesis (2766) was the largest groups, followed by post-translational modification, protein turnover, and chaperon $(2,112)$ and general function prediction of function only $(1,801)$.

KEGG pathway database is a knowledge base for the systematic analysis of gene functions in terms of networks of genes and molecules in cells [26]. Based on KEGG, 21691 unigenes were assigned to 130 pathways (Table 2, Figure 3). The pathways involving the largest number of translations were carbohydrate metabolism $(3632,16.74 \%)$ and translation $(3117,14.37 \%)$. In contrast, membrane transport $(59,0.27 \%)$ was the smallest group (Figure $3 \mathrm{C})$.

\section{Identification of DEGs}

To reveal the molecular mechanism involved in flavone, flavanol, and flavonoid biosynthesis, the DEGs between ROB and RPL were identified. Pearson's correlation coefficient $\left(r^{2}\right)$ between samples was 
relatively high in ROB_1 vs ROB_2 $\left(r^{2}=0.880\right)$ and RPL_1 vs RPL_2 $\left(r^{2}=0.867\right)$, whereas it was low in ROB vs. RPL (from 0.527 to 0.576 ). Thus, we expected to obtain a large number of DEGs between ROB and RPL. Read counts of every gene obtained through Hi-seq in all four RNA-sequencing samples (two biological replicates in ROB and RPL, respectively) were normalized and analyzed via DE-Seq package, finally 19,242 genes were detected as significantly differentially expressed between ROB and RPL.

\section{Combined analysis of RNA-sequencing and metabolomics data}

Emodin was methylated by methyl-transferase, followed by methoxy to form physcion. According to the results of the metabolomics analysis, the content of emodin in ROB is slightly lower than that observed in RPL. In contrast, the content of physcion in ROB was approximately 2.4-fold higher than that reported in RPL (Figure 4B). Since the amount of physcion is different in ROB and RPL, we then searched possible enzyme genes which may contribute to this difference (Figure 4A). We mined 121 genes involved in flavonoid biosynthesis, of which 38 were detected as DEGs. Among 38 DEGs, 7 genes (cluster14354.36512, cluster-14354.38156, cluster-14354.46426, cluster-14354.26346, cluster-14354.3016, cluster-14354.37142, and cluster-14354.3014) were annotated with the activity of caffeoyl coenzyme methyltransferase and their expression levels are significantly different between leaves of ROB and RPL (Figure 4C). And we further found that four genes (cluster-14354.38156, cluster-14354.26346, cluster14354.3016 and cluster-14354.3014) were expressed in both ROB and RPL (Figure 4C).

We used RT-qPCR to quantify the four genes obtained from the above analysis. The results showed that the expression of the cluster-14354.38156 in the ROB was approximately 14 -fold higher than that observed in the RPL. This finding was consistent with the difference detected by RNA-seq gene expression. Therefore, cluster-14354.38156 may regulate the metabolic reaction of emodin 3 methylation to physcion (Figure 4D).

\section{Discussion}

Natural products including herbal varieties and animal, used very widely as foods and dietary supplements, are important economic commodities in all countries. Comprehensive metabolites is important not only for pharmacological valuation but also for quality control. For products obtained from species differentially present in different countries, the transcriptome approach can provide a very reliable means of gene discovered, which is based on an accurate quantitative analysis of the metabolites. Totally, 62 compounds simultaneously quantitated using a dynamic multiple reactions monitoring (MRM) mode under unit mass-resolution, among 21 anthraquinone compounds have upstream or downstream biosynthesis relations may have different medicinal values. A comprehensive and systematic metabonomics analysis is of great significance to utilization of medicinal value of characteristic compounds of the mixed used two rhubarb. 
The combination of functional gene expression and metabolomic data is a useful approach based on the principle that genes and metabolites involved in the same metabolic process exhibit identical or similar variation, but lack of genome information usually make analysis of functional gene difficult. Thus, transcriptome provide a valuable way to simultaneously investigate functional gene and metabolite. The difference in the content of metabolites among different plant varieties or tissues could be determined through metabolomics analysis, while the difference in gene expression was determined via transcriptome sequencing. Combined them together, differentially expressed genes involved in metabolic pathways are possible genes functioning in the process of metabolite synthesis. In this study we generated 180,689 transcripts and 90,242 unigenes by the de novo transcriptome assembly. GO and KEGG pathway analyses characterized the unigenes, resulting in 21,691 unigenes with metabolic pathway annotations. A total of 121 genes were annotated in the flavonoid pathways, and 38 of them showed a significant difference in expression level between the RPL and ROB. The metabolomics result showed that the content of emodin in ROB is slightly lower than that observed in RPL, however the content of physcion in ROB was approximately 2.4-fold higher than that reported in RPL. According to the difference in the content of emodin and physcion in the two kinds of rhubarb and the expression level of key enzyme genes, we further search 7 differentially expressed genes annotated with the activity of caffeoyl coenzyme methyltransferase. RT-qPCR results showed that the expression of the cluster14354.38156 in the ROB was approximately 14 -fold higher than that observed in the RPL, which may participate in the process of O-methylation from emodin to physcion. Our study showed that the combination analysis of transcriptome and metabolome can indeed help in the search for enzyme genes involved in metabolite biosynthesis.

\section{Conclusions}

In the present study, using two rhubard species (Rheum Palmatum L. and Rheum Officinale Bails), both of which do not have a reference genome, we performed metabolome analysis and transcriptome sequencing to search key genes. Based on the large-scale quantification of target metabolites combined with the transcriptome data of RPL and ROB, we search 7 differentially expressed genes annotated with the activity of caffeoyl coenzyme methyltransferase. Furthermore, RT-qPCR results sussgested that the cluster-14354.38156 may participate in the process of 0-methylation from emodin to physcion. Our study supplied a useful resource for further study of metabolite in rhubarb and provided further insight into the combination analysis of transcriptome and metabolome, which can indeed help in the search for enzyme genes involved in metabolite biosynthesis.

\section{Methods}

\section{Plant material and metabolism chemical}

Two rhubarb species, Rheum Palmatum L. (RPL) and Rheum Officinale Bails (ROB) used in the study, were cultivated under the same conditions at the same base of the wild breeding base of medicinal materials in Enwei Plateau, Kangding, Sichuan, China. The collected rhizomes specimens were 
sequenced, and the spliced gene sequences were subjected to blast alignment, identified as rhubarb. The percent identity were $98.12 \%$ and $98.13 \%$, respectively. Then they were morphologically identified as Rheum Palmatum L. (RPL) and Rheum Officinale Bails (ROB) by a taxonomist (Professor Li Xiang) at Institute of Chinese Materia Medica, China Academy of Chinese Medical Science, BeiJing, China. Experimental research on plants, including collection of plant material, complied with institutional, national, or international guidelines. And we comply with the Convention on the Trade in Endangered Species of Wild Fauna and Flora. Fresh leaves were collected in liquid nitrogen, and then stored at $-80^{\circ} \mathrm{C}$ for later analysis. Our experimental research on rhubarb, including collection of plant material, complied with institutional, national, or international guidelines. Gradient grades of methanol, acetonitrile, and acetic acid were purchased from Sigma-Aldrich (St. Louis, MO, USA). Formic acid (eluent additive used for HPLC-MS analysis) used was of MS grade (CNW, Germany). The remaining analytical-grade chemicals were obtained from Beijing Chemical Factory (Beijing, China). Pure standards of major anthraquinones (rhein (No.0757-9804), emodin (No.0756-9707), aloe-emodin (No.110795-201007), chrysophanol (No.110796-201319), physcion (No.0758-9402) (> 98\%), and saikosaponin A (Internal standard, IS)) were purchased from the National Institute for the Control of Biological and Pharmaceutical Drugs (Beijing, China). Emodin-8-0- $\beta-D-g l u c o s i d e ~(>97.6 \%)$ was obtained from the laboratory where the study was conducted.

\section{Sample preparation and extraction}

Accurately weighed about $100 \mathrm{~g}$ rhein fresh leaf crushed using a mixer mill with a zirconia bead for 1.5 min at $30 \mathrm{~Hz}$, then extracted overweight at $4^{\circ} \mathrm{C}$ with $1.0 \mathrm{ml}$ pure methanol (or $70 \%$ aqueous methanol) containing $0.1 \mathrm{ppm}$ lidocaine for lipid-solubility extracts were absorbed and $0.4 \mathrm{ml}$ of each extract was mixed and filtrated (SCAA-104, $0.22 \mu \mathrm{m}$ pore size) before LC-MS analysis.

\section{ESI-Q-TOF-MS/MS}

The analytical conditions were as follows, UPLC: column, Agilent Elipse Plus $\mathrm{C}_{18}$ column (pore size 1.8 $\mu \mathrm{m}$, length $2.1 \times 50 \mathrm{~mm})$; solvent system, water $(0.1 \%$ formic acid), acetonitrile $(0.1 \%$ formic acid); gradient program, 100:0 V/V at $0 \mathrm{~min}, 5: 95 \mathrm{~V} / \mathrm{V}$, at $20.0 \mathrm{~min}, 5: 95 \mathrm{~V} / \mathrm{V}$ at $22.0 \mathrm{~min}, 95: 5 \mathrm{~V} / \mathrm{V}$ at $22.1 \mathrm{~min}$, 95:5 V/V at $28 \mathrm{~min}$; flow rate, $0.2 \mathrm{~mL} / \mathrm{min}$; temperature, $40^{\circ} \mathrm{C}$; injection volume: $1 \mu$ l. The effluent was alternatively connected to an ESI-triple quadruple-linear ion trap (Q-Trap)-MS or ESI-Q-TOF-MS.

Full time-of-flight (TOF) scans of UPLC effluents were acquired in the mass range of 50-1000 using an Agilent 6540-mass time-of-flight (Q-TOF) mass spectrometry equipped with a Dual ESI electrospray ion source in both positive and negative mode. The ESI source operation parameters, operating in a positive ion mode and the ESI-MS conditions are as followed: Gas temp: $325^{\circ} \mathrm{C}$, Gas Flow (L/min): 5 , Nebulizer (psig): 35 , Sheath Gas Temp: $350^{\circ} \mathrm{C}$, collision-energy voltage: $20 \mathrm{~V}(\mathrm{ESI}+)$ and $30 \mathrm{~V}(\mathrm{ESI}+)$ as well as $40 \mathrm{~V}$ 
(ESI+). Internal references (Purine and HP-0921) were adopted to modify the measured masses in real time, and the reference masses in positive ion mode were at $\mathrm{m} / \mathrm{z} 121.0509$ and 922.0098 , while in negative ion mode, they were at $\mathrm{m} / \mathrm{z} 119.0363$ and 1033.9881 .

\section{ESI-Q-TRAP-MS/MS}

A triple quadrupole-linear ion trap mass spectrometer (Q-trap), Q-TRAP 5500 LC-MS/MS system, equipped with an ESI-Turbo Ion-Spray interface, operating in a positive and negative mode by Analyst 1.6.2 software (AB Sciex). The ESI source operation parameters were as followed: source temperature $500^{\circ} \mathrm{C}$, ion spray voltage (IS) $5500 \mathrm{~V}$; ion source gas I (GS I), gas II (GS II), collision gas (CAD), curtain gas (CUG) was set at 50, 50, and 30 psi, respectively. Collision energy was also set at 20, 30 and $40 \mathrm{eV}$. Instrument running and mass calibration were performed with 1,10 and $100 \mathrm{~mol} / \mathrm{L}$ polypropylene glycol solutions in trap and LIP modes, respectively. The MIM scan which served as a survey scan to trigger informationdependent acquisition (IDA) of EPI scan model, MIM-EPI was carried out to screen metabolites ions isolated in Q1 mininal CE (5 eV). The corresponding Q3 at the same metabolites were monitored through two ways. The firstly, a modified MIM-EPI scan was adapted, in which Q1 and Q3 were set from 50.1 to 1000.0 Da, and the mass step was 0.2 Da. At the same time, the other target-scan was also acquired for paired Q1 and Q3. We monitored each MIM-EPI experiment with $60 \mathrm{MIM}$ transitions, product ions of each metabolites ion were scanned from 50.1 to $1000.0 \mathrm{Da}$ in Q3, and the total cycle time for one scan was approximately $2.0 \mathrm{~s}$.

\section{QQQ-Experiment}

The sample extracts were analyzed using UPLC-ESI-QQQ-MS (Agilent 1290 and 6460 triple quadrupole mass spectrometry series, Agilent Corporation, CA, USA). The quantitative data was acquired by QQQ scan using an improved Dynamic MRM experiments with collision gas (nitrogen) set to 5 psi. Improved of paired Q1-Q3 mass, CE, retention time, fragment for individual MRM transitions were done based on the EPI experiment scan and targeted scan. Each MRM transition was obtained with a 10-ms dell time and 10-ms pause time. The MS conditions in the positive mode were as follows: HV voltage 4000 kV; capillary $7 \mu \mathrm{A}$, nozzle voltage $500 \mathrm{~V}$; delta EMV $300 \mathrm{~V}$, gas flow $5 \mathrm{~L} / \mathrm{min}$; gas temperature $400{ }^{\circ} \mathrm{C}$; sheath gas flow $11 \mathrm{~L} / \mathrm{min}$. Collision energy was optimized based on the standards. Helium was used as the collision gas for collision-induced dissociation (CID). Quantification was done using the multiple reactions monitoring (MRM) mode under unit mass-resolution conditions.

The t-test was used for the quantitative analysis of compound results. Principal component analysis was performed on the metabolic data for both cultivars of rhubarb. 


\section{RNA extraction, library construction and sequencing}

Fresh leaves of the two cultivars were individually ground to powder in liquid nitrogen, each sample with two biological replicates. Total RNA was isolated using the RNA prep Pure Kit (For Plant TIANGEN Biotech, Beijing, China), and RNA degradation and contamination were determined using $1 \%$ agarose gels. RNA purity was verified using the Nano-Photometer ${ }^{\circledR}$ spectrophotometer (Implen, CA, USA). RNA concentration was measured using a Qubit ${ }^{\circledR}$ RNA Assay Kit in Qubit ${ }^{\circledR}$ 2.0 Fluorometer (Life Technologies, CA, USA). RNAintegrity was assessed using the RNA Nano 6000 Assay Kit on the Agilent Bioanalyzer 2100 system (Agilent Technologies, CA, USA).

A total amount of $1.5 \mu \mathrm{g}$ RNA per sample was used as input material for the RNA sample preparations. Sequencing libraries were generated using NEB Next ${ }^{\circledR}$ Ultra ${ }^{T M}$ RNA Library Prep Kit for Illumina ${ }^{\circledR}$ (NEB, USA) according to the recommendation of the manufacturer, and index codes were added to attribute sequences to each sample. The clustering of the index-coded samples was performed on a cBot Cluster Generation System using TruSeq PE Cluster Kit v3-cBot-HS (Illumina) according to the instructions provided by the manufacturer. After cluster generation, the library preparations were sequenced using an Illumina Hiseq 2500 platform and paired-end reads were generated.

\section{Sequence reads mapping, assembly and annotation}

Raw data in FASTQ format were firstly processed through in-house Perl scripts. Reads containing adapter sequences, Ns, and low-quality reads were excluded from the raw data. The remaining reads were kept as clean data. Subsequently, the Q20, Q30 and GC-content of the clean data were calculated. All the downstream analyses were based on clean data with high quality.

The FASTQ files from all four samples were pooled for the subsequent assembly. Transcriptome assembly was accomplished using the Trinity software assembly [22] with all parameters being set as default. All assembled contigs were then clustered to unigenes.

Gene function was annotated based on the following databases: $\mathrm{Nr}$ (National Center for Biotechnology Information (NCBI) non-redundant protein sequences), Nt (NCBI non-redundant nucleotide sequences), Pfam (Protein family) [27], EuKaryotic Orthologous Groups (KOG)/ Clusters of Orthologous Groups of proteins, Swiss-Prot (a manually annotated and reviewed protein sequence database), Kyoto Encyclopedia of Genes and Genomes (KEGG) Ortholog database, Gene Ontology (GO). Data for each sequenced library were analyzed using BLAST with a cutoff E-value of $10^{-5}$.

\section{Differential expression analysis of unigenes}


The levels of gene expression in each sample were estimated by RSEM [19]. Clean data were mapped back onto the assembled transcriptome and a read count for each gene was obtained using $\mathrm{Hi}$ sequencing. Differential expression analyses of two conditions/groups were performed using the DE-Seq $R$ package (1.10.1) [13]. We used count data generated by RSEM as input, two groups, i.e. two ROB samples and two RPL samples, were normalized and further used for differentially expression analysis by DE-seq data set from Matrix and DEseq functions in DE-Seq package. DE-Seq provided statistical routines for determining differential gene expression using a model based on the negative binomial distribution. The resulting $P$ values were adjusted and genes with an adjusted $P$-value $<0.05$ (according to DE-Seq) were assigned as differentially expressed.

\section{Combined analysis based on metabolome and the transcriptome}

We aimed to explore key enzyme genes involved in the biosynthesis pathway of anthraquinone. Since biosynthesis pathway of anthraquinone is poorly annotated, we searched the differentially expressed genes (DEGs) which involved in the flavonoid biosynthesis, which is well annotated. Then, according to the content difference of physcion in ROB and RPL, we picked up DEGs annotated as caffeoyl coenzyme methyltransferase as possible candidate of key enzyme involved in anthraquinone biosynthesis pathway. Since we have just two samples each group in DE analysis, we further used RT-qPCR to validate related DEGs. The primers were designed with Primer 5 software using the gene sequence obtained by RNAsequencing (Additional file 4). The primer design was based on common PCR primer design; however, the optimal length of the RT-qPCR primers was $80-180 \mathrm{bp}$, and the annealing temperature of the primers was $55-58^{\circ} \mathrm{C}$.

The RT-qPCR method is based on the specification of Trans-Start Green qPCR SuperMix UDG kit purchased from BeiJing all Gold Biotech Co. Ltd (Additional file 5). The template was diluted to approximately $1 \mu \mathrm{g} / \mu \mathrm{L}$. The qPCR reaction program involved incubation at $95^{\circ} \mathrm{C}$ for $2 \mathrm{~min}$, denaturation at $94^{\circ} \mathrm{C}$ for $15 \mathrm{~s}$, annealing at $60^{\circ} \mathrm{C}$ for $30 \mathrm{~s}$, extension at $72^{\circ} \mathrm{C}$ for $30 \mathrm{~s}$, and a total of 40 cycles.

\section{Abbreviations}

RPL: Rheum Palmatum L. ; ROB: Rheum Officinale Bails.

\section{Declarations}

\section{Ethics approval and consent to participate}

Not applicable. 
Not applicable.

\section{Availability of data and materials}

The datasets during and/or analysed during the current study available from the corresponding author on reasonable request.

\section{Competing interests}

The authors declare that they have no competing interests.

\section{Funding}

Our research was supported by "the Fundamental Research Funds for the Central public welfare research institutes" (No. ZZ13-YQ-057). The funding organization had no role in the design of the study, data collection, analysis, decision to publish the data, or preparation of the manuscript.

\section{Authors contributions}

SC conceived and designed the experiments; $J L$ and $L L$ performed the experiments and contributed to the acquisition of data; SC, JL and HG analyzed the data; SC and AL wrote the paper. All authors have revised the manuscript critically for important intellectual content and given final approval of the version to be published. SC are responsible for the integrity of the work as a whole.

\section{Acknowledgments}

The authors are grateful to Uni-edit (www.uni-edit.net) for editing and proofreading this manuscript.

\section{References}

1. Zheng QX, Hai-Feng WU, Guo J, Nan HJ, Chen SL, Yang JS, Xu-Dong XU: Review of Rhubarbs: Chemistry and Pharmacology. Chinese Herbal Medicines 2013, 5(1):9-32. 
2. Hu Y, Xie X, Wang L, Zhang H, Yang J, Li Y: Genetic variation in cultivated Rheum tanguticum populations. Genetics \& Molecular Biology 2014, 37(3):540-548.

3. Pharmacopoeia Committee of P.R. China E: Chinese Pharmacopoeia, Chemical Industry Publishing House, BeiJing 2015:P23-24.

4. Hao S, Luo G, Chen D, Zheng X: A Comprehensive and System Review for the Pharmacological Mechanism of Action of Rhein, an Active Anthraquinone Ingredient. Frontiers in Pharmacology 2016, 7.

5. Ye M, Han J, Chen $\mathrm{H}$, Zheng J, Guo D: Analysis of phenolic compounds in rhubarbs using liquid chromatography coupled with electrospray ionization mass spectrometry. J Am Soc Mass Spectr 2007, 18(1):82-91.

6. Koirala N, Pandey RP, Parajuli P, Jung HJ, Sohng JK: Methylation and subsequent glycosylation of 7,8-dihydroxyflavone. J Biotechnol 2014, 184:128-137.

7. Cohen DR, Townsend CA: A dual role for a polyketide synthase in dynemicin enediyne and anthraquinone biosynthesis. Nature Chemistry 2018, 10(2):231-236.

8. Eichinger D, Bacher A, Zenk MH, Eisenreich W: Quantitative Assessment of Metabolic Flux by $13 \mathrm{C}$ NMR Analysis. Biosynthesis of Anthraquinones in Rubia tinctorum. J Am Chem Soc 1999, 121(33):7469-7475.

9. Han YS, Heijden RVD, Verpoorte R: Biosynthesis of anthraquinones in cell cultures of the Rubiaceae. Plant Cell Tissue \& Organ Culture 2001, 67(3):201-220.

10. Pandith SA, Dar RA, Lattoo SK, Shah MA, Reshi ZA: Rheum australe , an endangered high-value medicinal herb of North Western Himalayas: a review of its botany, ethnomedical uses, phytochemistry and pharmacology. Phytochemistry Reviews 2018, 17(3):1-37.

11. Austin MB, Noel JP: The chalcone synthase superfamily of type III polyketide synthases. Cheminform 2003, 20(1):79-110.

12. Saito K, Yonekura-Sakakibara K, Nakabayashi R, Higashi Y, Yamazaki M, Tohge T, Fernie AR: The flavonoid biosynthetic pathway in Arabidopsis: Structural and genetic diversity. Plant Physiology \& Biochemistry 2013, 72(Complete):21-34.

13. Wang LK, Feng ZX, Wang X, Wang XW, Zhang XG: DEGseq: an R package for identifying differentially expressed genes from RNA-seq data. Bioinformatics 2010, 26(1):136-138.

14. Yuan X, Li K, Huo W, Lu X: De Novo Transcriptome Sequencing and Analysis to Identify Genes Involved in the Biosynthesis of Flavonoids in Abrus mollis Leaves. Russ J Plant Physl+ 2018, 65(3):333-344.

15. Marc S, Anne-Marie K, Rianne L, Vernooy JH, Wind JC, Wullems GJ, Croes AF: Regulation of anthraquinone biosynthesis in cell cultures of Morinda citrifolia. Journal of Plant Physiology 2003, 160(6):607-614.

16. Pandith SA, Dhar N, Rana S, Bhat WW, Kushwaha M, Gupta AP, Shah MA, Vishwakarma R, Lattoo SK: Characterization and functional promiscuity of two divergent paralogs of Type III plant polyketide synthases from Rheum emodi Wall ex. Meissn. Plant Physiology 2016, 171(4):2599-2619. 
17. Shkryl YN, Veremeichik GN, Bulgakov VP, Zhuravlev YN: Induction of anthraquinone biosynthesis in Rubia cordifolia cells by heterologous expression of a calcium-dependent protein kinase gene. Biotechnology \& Bioengineering 2011, 108(7):1734-1738.

18. Soták M, Czeranková O, Klein D, Jurčacková Z, Ling L, Čellárová E: Comparative Transcriptome Reconstruction of FourHypericumSpecies Focused on Hypericin Biosynthesis. Frontiers in Plant Science 2016, 7(R106).

19. Li XH, II Park N, Xu H, Woo SH, Park CH, Park SU: Differential Expression of Flavonoid Biosynthesis Genes and Accumulation of Phenolic Compounds in Common Buckwheat (Fagopyrum esculentum). J Agr Food Chem 2010, 58(23):12176-12181.

20. Osakabe Y, Liang ZC, Ren C, Nishitani C, Osakabe K, Wada M, Komori S, Malnoy M, Velasco R, Poli M et al: CRISPR-Cas9-mediated genome editing in apple and grapevine. Nature Protocols 2018, 13(12):2844-2863.

21. 21. Chen S, Xiang Y, Deng J, Liu YL, Li SH: Simultaneous Analysis of Anthocyanin and NonAnthocyanin Flavonoid in Various Tissues of Different Lotus (Nelumbo) Cultivars by HPLC-DAD-ESIMS(n.). PloS one 2013, 8(4):e62291-e62291.

22. Zheng Z, Li S, Zhong Y, Zhan R, Yan Y, Pan H, Yan P: UPLC-QTOF-MS Identification of the Chemical Constituents in Rat Plasma and Urine after Oral Administration of Rubia cordifolia L. Extract. Molecules 2017, 22(8).

23. 23. Grabherr MG, Haas BJ, Yassour M, Levin JZ, Thompson DA, Amit I, Adiconis X, Fan L, Raychowdhury R, Zeng QD et al: Full-length transcriptome assembly from RNA-Seq data without a reference genome. Nat Biotechnol 2011, 29(7):644-U130.

24. 24. Altschul SF, Madden TL, Sch?Ffer AA, Zhang J, ., Zhang Z, ., Miller W, ., Lipman DJ: Gapped BLAST and PSI-BLAST: a new generation of protein database search programs. 1997.

25. 25. Consortium TGO: Gene Ontology Consortium: going forward. Nucleic Acids Res 2015, 43(Database issue):1049-1056.

26. 26. Kanehisa $M$, Goto $S$, Sato $Y$, Kawashima M, Furumichi $M$, Tanabe M: Data, information, knowledge and principle: back to metabolism in KEGG. Nucleic Acids Res 2014, 42(D1):D199-D205.

27. El-Gebali S, Mistry J, Bateman A, Eddy SR, Luciani A, Potter SC, Qureshi M, Richardson LJ, Salazar GA, Smart A et al: The Pfam protein families database in 2019. Nucleic Acids Res 2019, 47(D1):D427-D432.

\section{Tables}

Table 1. Qualitative result of metabolites in rhubarb leaves 
MS

\begin{tabular}{|c|c|c|c|c|c|}
\hline \multirow[t]{21}{*}{ Anthraquinone } & 1 & 8-O-Methylchrysophanol & 5.51 & {$[\mathrm{M}+\mathrm{H}]+291$} & 139 \\
\hline & 2 & Physcion-O-glucoside & 7.51 & {$[\mathrm{M}-\mathrm{H}]-445$} & 283 \\
\hline & 3 & Rhein-O-glucoside & 7.73 & {$[\mathrm{M}-\mathrm{H}]-445$} & 239 \\
\hline & 4 & Chrysophanol-8-O-(6’-O-malonyl)-glucoside & 7.88 & {$[\mathrm{M}-\mathrm{H}]-567$} & 169 \\
\hline & 5 & Physcion-8-o-- $\beta$-d-(6'-acetyl)-glucoside & 8.30 & {$[\mathrm{M}-\mathrm{H}]-487$} & 283 \\
\hline & 6 & Emodin-O-glucoside & 8.68 & {$[\mathrm{M}-\mathrm{H}]-431$} & 269 \\
\hline & 7 & Chrysophanol-O-glucoside & 8.81 & {$[\mathrm{M}-\mathrm{H}]-415$} & 253 \\
\hline & 8 & Emodin-O-(6`-O-acetyl)-glucoside & 9.06 & {$[\mathrm{M}-\mathrm{H}]-473$} & 269 \\
\hline & 9 & Emodin-8-O-(6`-O-malonyl)-glucoside & 9.07 & {$[\mathrm{M}-\mathrm{H}]-517$} & 473 \\
\hline & 10 & Chrysophanol-O-(6'-acetyl)-glucopyranoside & 9.46 & {$[\mathrm{M}-\mathrm{H}]-457$} & 253 \\
\hline & 11 & Rhein-1-O-(O-acetyl)-glucoside & 9.85 & {$[\mathrm{M}-\mathrm{H}]-487$} & 239 \\
\hline & 12 & Citreorosein & 10.21 & {$[\mathrm{M}-\mathrm{H}]-285$} & 211 \\
\hline & 13 & Aloe emodin & 10.33 & {$[\mathrm{M}-\mathrm{H}]-269$} & 240 \\
\hline & 14 & Rhein & 11.45 & {$[\mathrm{M}-\mathrm{H}]-283$} & 239 \\
\hline & 15 & Emodin & 11.72 & {$[\mathrm{M}-\mathrm{H}]-269$} & 225 \\
\hline & 16 & Chrysophanol & 13.10 & {$[\mathrm{M}-\mathrm{H}]-253$} & 225 \\
\hline & 17 & Physcion & 13.28 & {$[\mathrm{M}-\mathrm{H}]-283$} & 240 \\
\hline & 18 & Emodin bianthrones B + C3H2 & 13.54 & {$[\mathrm{M}-\mathrm{H}]-547$} & 254 \\
\hline & 19 & Emodin bianthrones B & 14.03 & {$[\mathrm{M}-\mathrm{H}]-509$} & 254 \\
\hline & 20 & Emodin bianthrones $\mathrm{B}+\mathrm{CH} 2 \mathrm{COOH}$ & 14.16 & {$[\mathrm{M}-\mathrm{H}]-568$} & 254 \\
\hline & 21 & Rheidin A & 16.13 & {$[\mathrm{M}-\mathrm{H}]-523$} & 254 \\
\hline \multirow[t]{5}{*}{ flavonoids } & 22 & Kaempferol & 5.29 & {$[\mathrm{M}+\mathrm{H}]+287$} & 213 \\
\hline & 23 & Quercetin-3-O-[acetyl-galactoside] & 5.55 & {$[\mathrm{M}+\mathrm{H}]+465$} & 345 \\
\hline & 24 & 12-(3-Hydroxyethyl)-cytisine & 6.10 & {$[\mathrm{M}+\mathrm{H}]+235$} & 189 \\
\hline & 25 & Myricetin-3-O- $\beta$-D-glucoside & 6.25 & {$[\mathrm{M}+\mathrm{H}]+481$} & 319 \\
\hline & 26 & Isovitexin & 6.54 & {$[\mathrm{M}+\mathrm{H}]+433$} & 283 \\
\hline
\end{tabular}




\begin{tabular}{|c|c|c|c|c|c|}
\hline & 27 & Hyperin & 6.66 & {$[\mathrm{M}+\mathrm{H}]+465$} & 303 \\
\hline & 28 & Quercetin & 6.67 & {$[\mathrm{M}+\mathrm{H}]+303$} & 153 \\
\hline & 29 & $\begin{array}{l}\text { Quercetin-3-O-[6"-(3-hydroxy-3-methylglutaroyl)- } \\
\text { galactoside] }\end{array}$ & 6.85 & {$[\mathrm{M}+\mathrm{H}]+609$} & 303 \\
\hline & 30 & 2"-O-galloylvitexin & 6.85 & {$[\mathrm{M}+\mathrm{H}]+585$} & 313 \\
\hline & 31 & Quercetin-3-Glucuronide+CH2 & 7.76 & {$[\mathrm{M}+\mathrm{H}]+477$} & 303 \\
\hline & 32 & Luteolin & 7.00 & {$[\mathrm{M}+\mathrm{H}]+287$} & 153 \\
\hline & 33 & 4'-Hydroxy-5,7-dimethoxyflavanone & 7.17 & {$[\mathrm{M}+\mathrm{H}]+463$} & 181 \\
\hline & 34 & Aphonol & 7.49 & {$[\mathrm{M}+\mathrm{H}]+433$} & 287 \\
\hline & 35 & Rutin & 6.41 & {$[\mathrm{M}+\mathrm{H}]+611$} & 303 \\
\hline & 36 & Naringenin & 9.82 & {$[\mathrm{M}+\mathrm{H}]+273$} & 153 \\
\hline & 37 & Apigenin & 13.31 & {$[\mathrm{M}-\mathrm{H}]-269$} & 151 \\
\hline & 38 & Pinobaksin & 14.45 & {$[\mathrm{M}+\mathrm{H}]+255$} & 153 \\
\hline stilbenes & 39 & Resveratrol-glucopyranoside & 7.30 & {$[\mathrm{M}-\mathrm{H}]-389$} & 227 \\
\hline & 40 & Rhapontigenin-(6`-O-acetyl)-glucopyranoside & 7.40 & {$[\mathrm{M}-\mathrm{H}]-461$} & 257 \\
\hline & 41 & Rhapontigenin & 7.60 & {$[\mathrm{M}-\mathrm{H}]-257$} & 215 \\
\hline & 42 & Rhapontigenin-glucopyranoside & 7.65 & {$[\mathrm{M}-\mathrm{H}]-419$} & 257 \\
\hline & 43 & Resveratrol-(6`-O-galloyl)-glucopyranoside & 7.83 & {$[\mathrm{M}-\mathrm{H}]-541$} & 313 \\
\hline & 44 & Piceatannol-O- $\beta$-D-glucopyranoside & 8.52 & {$[\mathrm{M}-\mathrm{H}]-405$} & 243 \\
\hline galloyl esters & 45 & Galloyl-glucose & 2.76 & {$[\mathrm{M}-\mathrm{H}]-331$} & 125 \\
\hline & 46 & Gallic acid & 3.11 & {$[\mathrm{M}-\mathrm{H}]-169$} & 125 \\
\hline & 47 & di-O-galloyl-glucose & 5.23 & {$[\mathrm{M}-\mathrm{H}]-483$} & 169 \\
\hline & 48 & p-coumaroyl-O-galloyl-glucose & 6.20 & {$[\mathrm{M}-\mathrm{H}]-477$} & 313 \\
\hline & 49 & tri-O-galloyl-glucose & 6.27 & {$[\mathrm{M}-\mathrm{H}]-635$} & 465 \\
\hline & 50 & p-coumaroyl-di-O-galloyl-glucose & 7.67 & {$[\mathrm{M}-\mathrm{H}]-629$} & 169 \\
\hline & 51 & p-coumaroyl-O-p-hydroxybenzoyl-galloyl-glucose & 8.02 & {$[\mathrm{M}-\mathrm{H}]-597$} & 433 \\
\hline & 52 & Cinnamoyl-O-galloyl-glucose & 8.08 & {$[\mathrm{M}-\mathrm{H}]-461$} & 125 \\
\hline & 53 & di-coumaroyl-O-galloyl-glucose & 8.36 & {$[\mathrm{M}-\mathrm{H}]-623$} & 459 \\
\hline & 54 & Coumaroyl-O-feruoyl-O-galloyl-glucose & 8.49 & {$[\mathrm{M}-\mathrm{H}]-653$} & 489 \\
\hline
\end{tabular}




\begin{tabular}{|c|c|c|c|c|c|}
\hline & 55 & Cinnamoyl-di-O-galloyl-glucose & 8.63 & {$[\mathrm{M}-\mathrm{H}]-613$} & 169 \\
\hline & 56 & Coumaroyl-O-cinnamoyl-O-galloyl-glucose & 9.44 & {$[\mathrm{M}-\mathrm{H}]-607$} & 443 \\
\hline \multirow[t]{3}{*}{ tanins } & 57 & Catechin-glucopyranoside & 4.93 & {$[\mathrm{M}+\mathrm{H}]+453$} & 139 \\
\hline & 58 & (-)-Epicatechin gallate & 7.17 & {$[\mathrm{M}-\mathrm{H}]-441$} & 169 \\
\hline & 59 & $(+)$-Catechin & 7.19 & {$[\mathrm{M}-\mathrm{H}]-289$} & 255 \\
\hline Naphthylenes & 60 & Torachrysone-8-O-ß-D-glucoside & 6.19 & {$[\mathrm{M}+\mathrm{H}]+409$} & 247 \\
\hline Alkaloids & 61 & Ephedrine & 4.59 & {$[\mathrm{M}+\mathrm{H}]+166$} & 77 \\
\hline Coumarin & 62 & 3(2'-Chlorophenyl)-7-ethoxycoumarin & 7.14 & {$[\mathrm{M}+\mathrm{H}]+463$} & 217 \\
\hline
\end{tabular}

Table 2. Summary of de novo sequence assembly for rhubarb leaves

\begin{tabular}{llllllll}
\hline Sample & Raw Reads & Clean Reads & Clean Bases & Error & Q20 & Q30 & GC Content \\
& & & & $(\%)$ & $(\%)$ & $(\%)$ & $(\%)$ \\
\hline ROB_1 & 51367574 & 48795386 & $7.32 G$ & 0.02 & 95.65 & 89.28 & 47.98 \\
ROB_2 & 49305010 & 46904390 & $7.04 G$ & 0.02 & 95.78 & 89.56 & 48.19 \\
\hline RPL_1 & 45103294 & 43417756 & $6.51 G$ & 0.02 & 96.93 & 92.27 & 48.98 \\
\hline RPL_2 & 48097370 & 45563138 & $6.83 G$ & 0.02 & 95.42 & 88.84 & 47.79 \\
\hline
\end{tabular}

Notes: a: ROB, Rheum palmatum L.; b: RPL, Rheum palmatum L.

Figures 


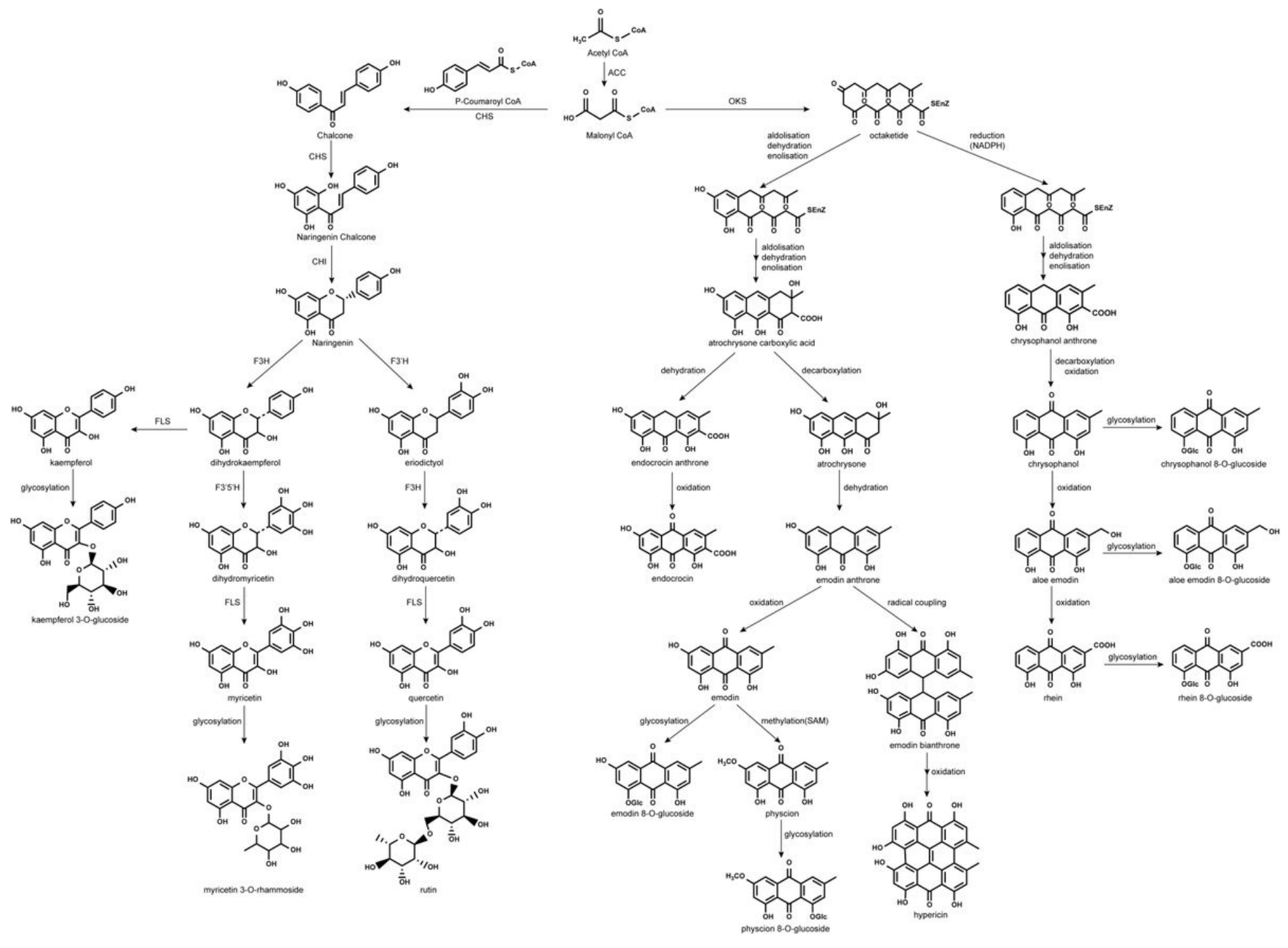

Figure 1

The biosynthetic metabolic network of target metabolites in rhubarb has been constructed. 

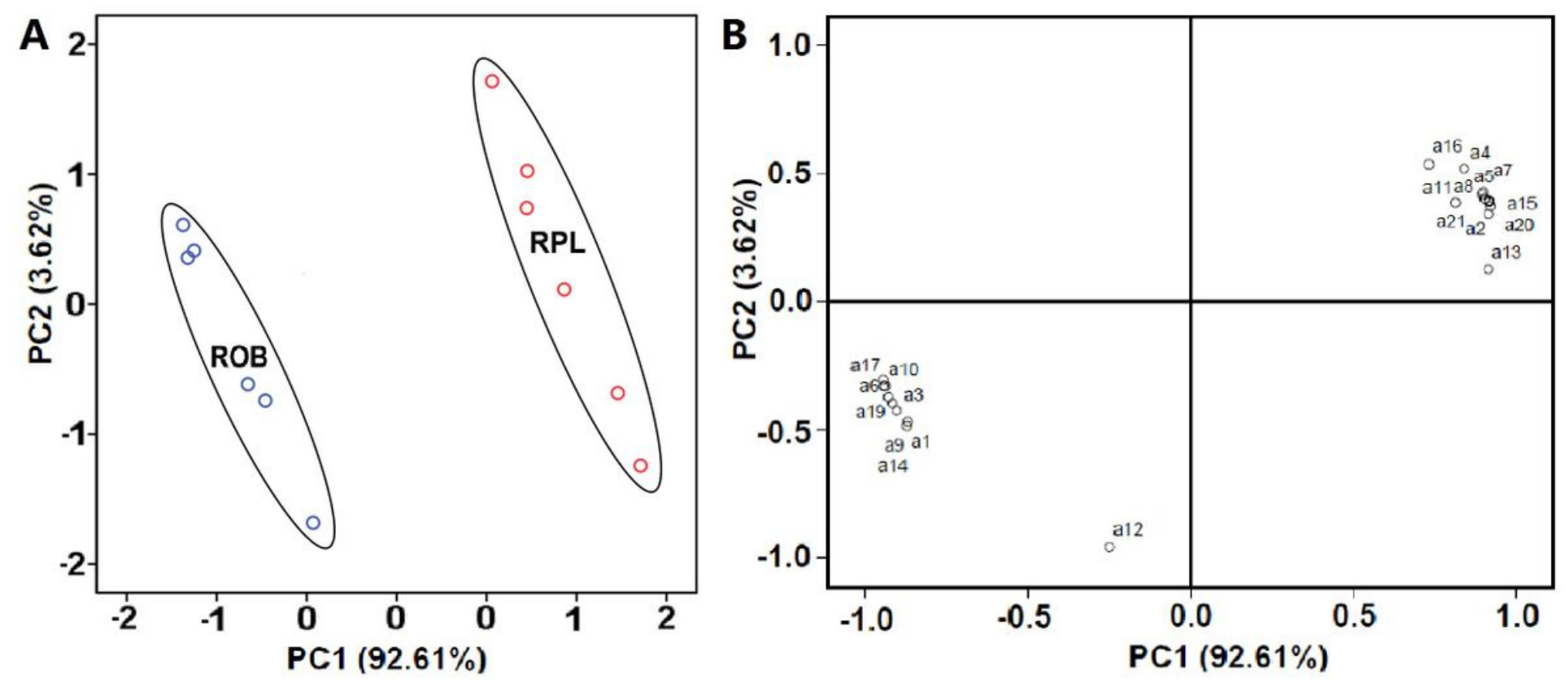

Figure 2

Positions of principal components analysis (PCA) scores (PC1, PC2) of the detected compounds in two rhubarbs. Notes: Percentages in the parentheses represent principal component variance. The number in the figure B represent the compound number, which correspond to the same compounds as in Table 1. A: Scores scatter plot of PCA, B: loadings plot of PCA. 

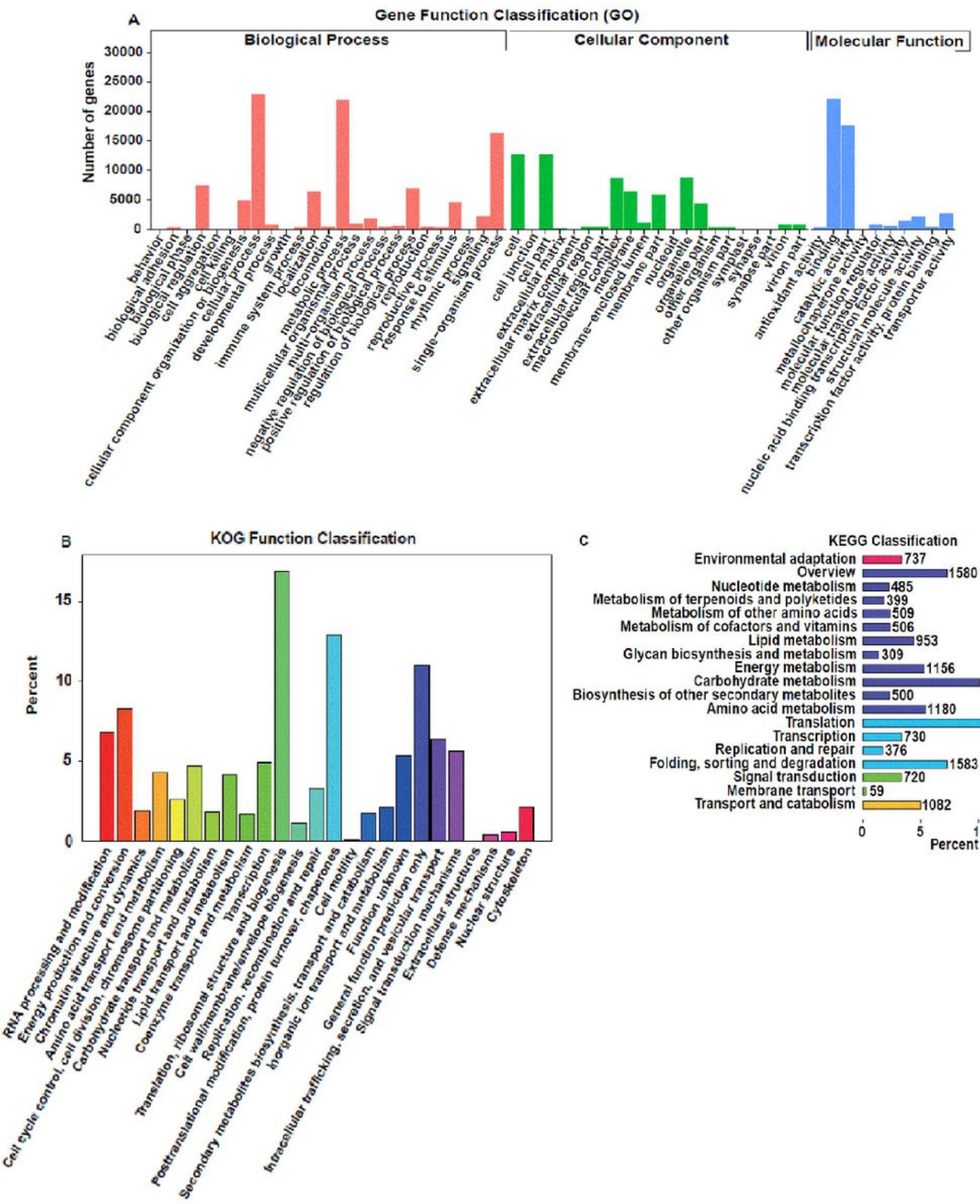

C
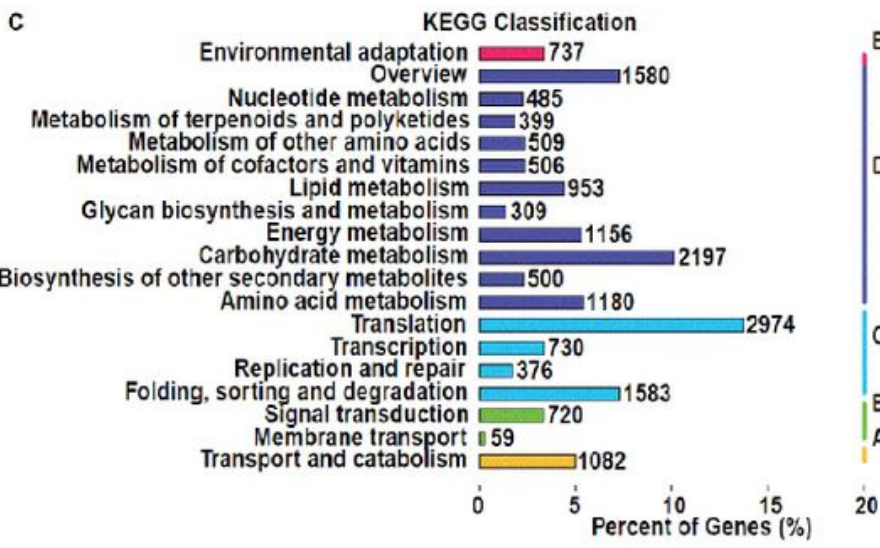

\section{Figure 3}

Functional categorization of unigens determined from three biological processed classification. A: Gene Ontology (Go). B: EuKaryotic Orthologous Groups (KOG). C: Kyoto Encyclopaedia of Genes and Genomes (KEGG). 
A

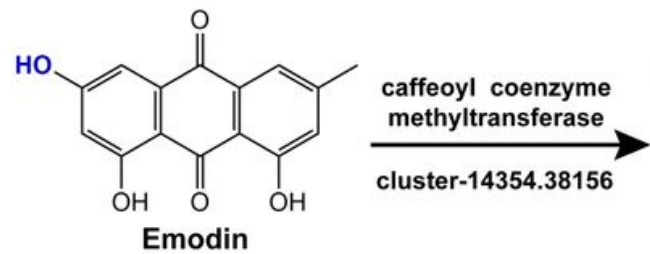

C

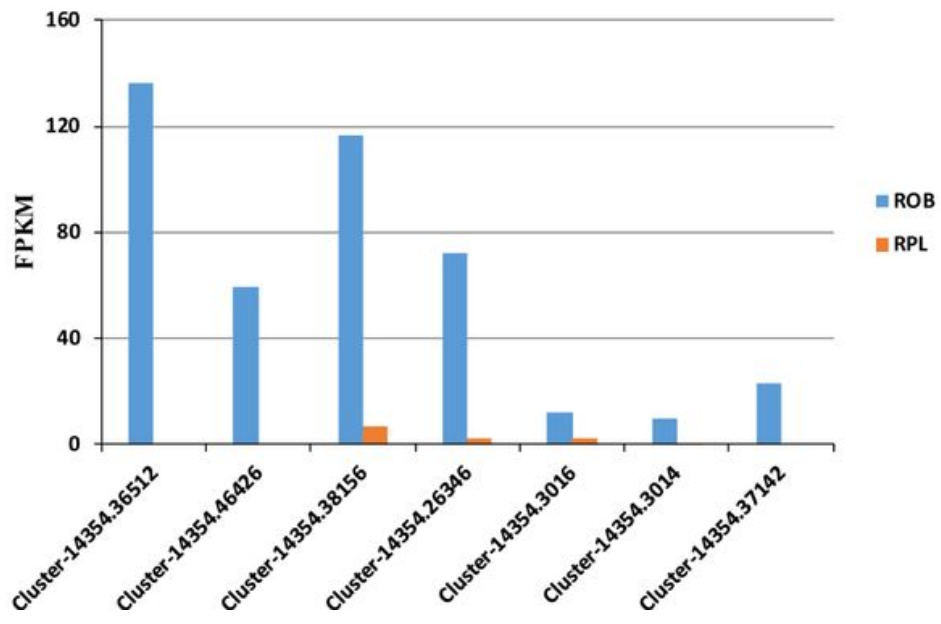

B
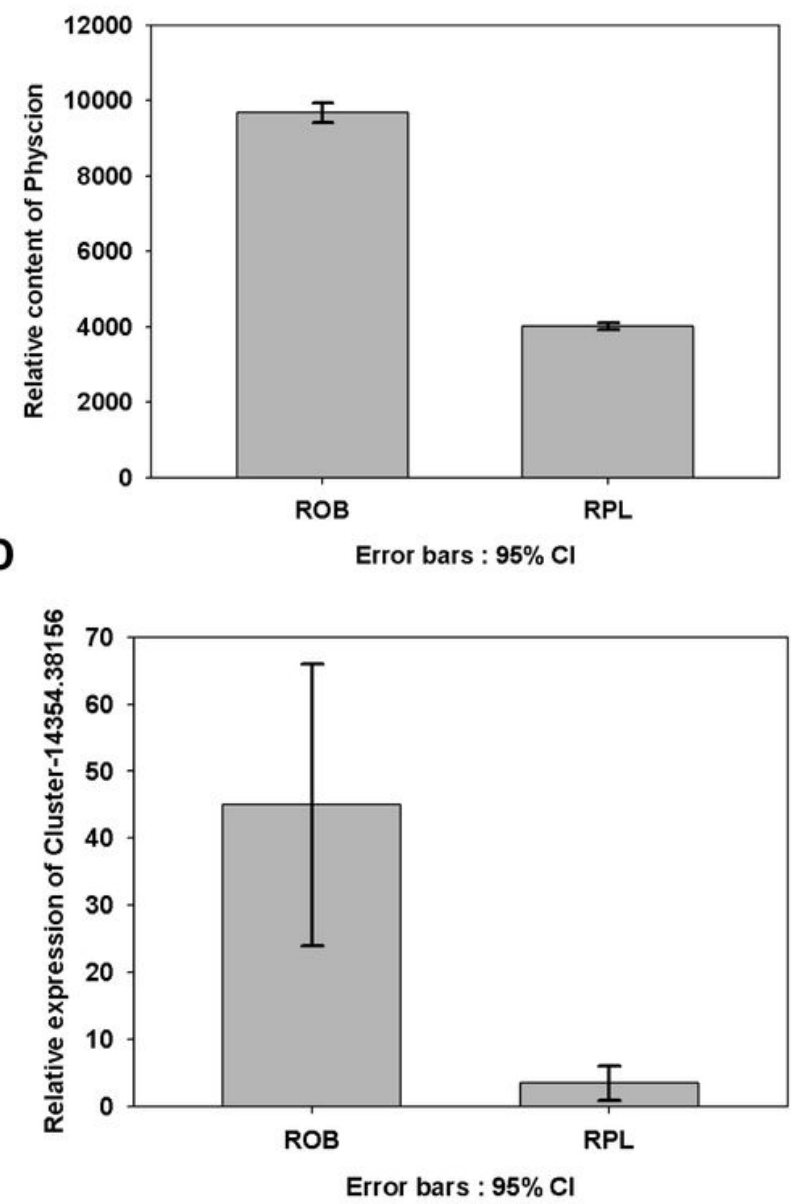

Figure 4

Candidate gene cluster-14354.38156 may be leading emodin to physcion (part A), Relative contents of physcion in two kinds of rhubarb (part B), difference expression level of seven selected genes (part C), related gene relative expression in the two kinds of rhubarb (part D).

\section{Supplementary Files}

This is a list of supplementary files associated with this preprint. Click to download.

- GraphicalAbsract.tif

- Additionalfiles.docx 